\title{
Overall survival of patients with EGFR mutation- positive non-small-cell lung cancer treated with erlotinib, gefitinib or afatinib under drug programmes in Poland - real-world data
}

\author{
Melania Brzozowska ${ }^{1}$, Waldemar Wierzba², Sylwia Szafraniec-Buryło ${ }^{3}$, Marcin Czech ${ }^{3}$, \\ Gabriela Majkut ${ }^{4}$, Joanna Połowinczak-Przybyłek ${ }^{5}$, Piotr Potemski ${ }^{6}$, Andrzej Śliwczyński ${ }^{1}$
}

\author{
${ }^{1}$ Division of Quality Services, Procedures and Medical Standards, Medical University \\ of Lodz, Lodz, Poland \\ 2UHE Satelite Campus in Warsaw, University of Humanities and Economics in Lodz, \\ Poland \\ ${ }^{3}$ Department of Pharmacoeconomics, Institute of Mother and Child, Warsaw, Poland \\ ${ }^{4}$ Analysis and Strategy Department, Central Office, National Health Fund, Warsaw, \\ Poland \\ ${ }^{5}$ Chemotherapy Department, Copernicus Memorial Hospital, Medical University \\ of Lodz, Lodz, Poland \\ ${ }^{6}$ Comprehensive Cancer Center and Traumatology, Copernicus Memorial Hospital, \\ Medical University of Lodz, Lodz, Poland
}

Submitted: 21 June 2018; Accepted: 13 November 2018

Online publication: 22 January 2019

Arch Med Sci 2021; 17 (6): 1618-1627

DOI: https://doi.org/10.5114/aoms.2018.80362

Copyright (c) 2018 Termedia \& Banach

\begin{abstract}
Introduction: The aim of the study was to estimate the overall survival of patients with EGFR mutation-positive non-small-cell lung cancer treated with erlotinib, gefitinib or afatinib.

Material and methods: Real-world patients who received afatinib, erlotinib or gefitinib between 1 July 2012 and 30 October 2017 were analysed in five subgroups.

Results: Among 267 patients treated with afatinib financed as the first line of treatment, 76 (28.46\%) deaths occurred. Median observation time was 12.8 months (95\% Cl: $11.2-13.9)$. Median OS was 22.8 months (95\% Cl: 19.2-27.1). Among 83 patients who received erlotinib financed exclusively as the second line of treatment the number of deaths was 74 (89.16\%). Median observation time was 64.3 months ( $95 \% \mathrm{Cl}$ : $60.4-64.6)$. Median OS was 16 months (95\% Cl: 13.2-22.9). Among 622 patients who received erlotinib financed both as first and second line treatment, there were 400 (64.3\%) deaths. Median observation time was 33.3 months (95\% Cl: 31.2-37.6). Median OS was 17.8 months ( $95 \% \mathrm{Cl}: 16.4-19.7)$. Among 137 patients who received gefitinib financed only as the first line of treatment, there were 128 (93.4\%) deaths. Median observation time was 58.3 months $(95 \% \mathrm{Cl}$ : 49.4-62.5). Median OS was 16 months (95\% Cl: 13.8-19.7). Among 348 patients who received gefitinib financed both as the first and second line of treatment the number of deaths was 208 (59.8\%). Median observation time was 23.7 months (95\% Cl: 20.7-28.7). Median OS was 15.5 months (95\% Cl: 12.9-17.5).

Conclusions: Our real-world data regarding OS confirm the benefits found in clinical trials from the use of afatinib, erlotinib or gefitinib. However, the lower overall survival rate of Polish patients compared to similar studies from other research centres suggests the need for deeper investigation of this issue.
\end{abstract}

Key words: erlotinib, gefitinib, afatinib, lung cancer, real-world data.

\author{
Corresponding author: \\ Gabriela Majkut MD, PhD \\ Analysis and \\ Strategy Department \\ Central Office \\ National Health Fund \\ 2 Hankiewicza St \\ 02-103 Warsaw, Poland \\ E-mail: gabriela.majkut@nfz. \\ gov.pl
}




\section{Introduction}

Lung cancer remains the leading cause of death worldwide among all cancers. The dismal 5 -year survival rate of $16 \%$ is in part due to the lack of symptoms during the early stages and lack of an effective screening test until recently. Subsequently, a number of studies compared computed tomography (CT) with the chest X-ray. These studies did identify lung cancer in earlier stages. However, they were not designed to prove a reduction in mortality. Later trials have focused on low-dose CT (LDCT) as a screening tool. Nonetheless, while a role for LDCT in lung cancer screening has been established, the issues of high false positive rates, radiation risk, and cost effectiveness still need to be addressed [1].

In parallel to the search for a test that can detect lung cancer at an early stage, the search for effective and cost-effective treatment regimes is ongoing, including real-world data as an additional tool for making clinical decisions. The largest source of such data in Poland is the National Health Fund, the public institution financing the drug programmes.

Drug programmes (DPs) are the way of governmental funding of new and costly licenced drugs in Poland for real-world patient populations with selected oncologic or non-oncologic diseases. Descriptions of DPs are published by Polish Minister of Health as a formal announcement. The description of each DP contains inclusion and exclusion criteria which are prepared based on risk stratification and cluster assignment of the patient population with each disease and allowance of enrolment and funding only for selected groups of patients chosen from the patient population in a non-randomized, non-controlled manner (as shown in Appendix). DPs also specify details on posology and indispensable methods of therapy monitoring. All insured patients in the whole country who are potential candidates for the treatment in routine clinical practice and fulfilling criteria defined are considered eligible. Unified patient inclusion criteria for the entire country allow for obtaining a highly homogeneous cohort of treated patients, which simplifies clinical effectiveness analyses and makes the DP population somewhat similar to the one enrolled in clinical trials.

Non-small cell lung cancer (NSCLC) therapy DPS have been available in Poland since 1 July 2012. Initially, gefitinib was intended for $1^{\text {st }}$ line and erlotinib for $2^{\text {nd }}$ line patients. Later their use was extended to $2^{\text {nd }}$ and $1^{\text {st }}$ line, respectively, and additionally the programme containing afatinib for $1^{\text {st }}$ line treatment was included in public financing from 1 May 2015. The basic molecular criterion which enabled patients' inclusion in erlotinib, gefitinib or afatinib DPs was the presence of an ac- tivating mutation of the epidermal growth factor receptor (EGFR) gene.

The objective of this study was to assess the overall survival of EGFR-mutation positive NSCLC patients treated with afatinib, erlotinib or gefitinib in DPs in Poland.

\section{Material and methods}

The Polish public payer database was used to extract data concerning lung cancer treatment services provided to patients (ICD-10 C34.\%, where the '\%' mark replaces any number). The database of the National Health Fund (Narodowy Fundusz Zdrowia - NFZ), the sole Polish public payer, contains data on all health services provided to Polish patients financed from public sources.

Based on a unique patient identifier (PESEL number) and respective DP identifiers, all dates of dispensing the drug to the patients participating in afatinib, erlotinib or gefitinib DPs were obtained. The dates of death were downloaded from the PESEL database. Complete observations refer to the cases with death date provided. Censored observations refer to the cases when there is no patient death date present in the databases within the analysed period (the patient is alive until the end of the observation period, that is until 30 October 2017).

The median overall survival (OS) for patients was calculated using the Kaplan-Meier estimator and survival tables. For each drug, the survival of patients was analysed, from the date of first administration of the respective drug (initial date) until 30 October 2017 (established cut-off date). Median observation time was calculated based on the censored observation time. To prepare the results of statistical analyses, the SAS E.G., v. 5.1 (SAS Institute Inc., Cary, North Carolina, USA) software was used. The difference in OS was assessed by a two-sided log-rank test, assuming statistical significance at $p$ less than 0.05 .

The population analysed was divided into five subgroups, for which the estimations were performed.

The population treated with afatinib was homogeneous, and only one group of patients was created as the drug was reimbursed in DP only in the $1^{\text {st }}$ line of treatment over the entire observation period of 30 months (2.5 years) that is, from 1 May 2015 until the cut-off date.

Erlotinib was available in DPs for 64 months (5.3 years) - from 1 July 2012 until the cut-off date. Two subgroups of patients in the population treated with erlotinib were created. The first erlotinib subgroup consists of patients who received erlotinib therapy reimbursement exclusively in the $2^{\text {nd }}$ line of treatment from 1 July 2012 to 1 March 2013. The second erlotinib subgroup consists of 
patients who commenced the treatment between 1 March 2013 and the cut-off date, at which time the medication was financed as part of the programme both as $1^{\text {st }}$ and $2^{\text {nd }}$ line treatment.

Gefitinib therapy was reimbursed under DPs for the same period as erlotinib - 64 months (5.3 years) - from 1 July 2012 until the cut-off date. Two gefitinib subgroups existed in the analysed population: patients included in the first subgroup initiated gefitinib therapy financed only as the $1^{\text {st }}$ line of treatment during the period from 1 July 2012 to 1 March 2014. The second gefitinib subgroup consists of patients receiving gefitinib under a DP both as the $1^{\text {st }}$ or $2^{\text {nd }}$ line of treatment. These patients initiated gefitinib therapy from 1 March 2014.

\section{Statistical analysis}

The method of descriptive statistical analysis was used, and the estimation of the survival curve was created using the Kaplan-Meier estimator. Due to the separate estimation of overall survival (OS) for each subpopulation, the comparison of the results of the estimation using statistical tests was waived.

\section{Results}

The afatinib subgroup ("afatinib" label in Figure 1) consisted of 267 patients: 99 (37.1\%) males and 168 (62.9\%) females. Median males' age was $64 \pm 10.1$ years (mean: 61.5); median females' age was $64 \pm 12.3$ years (mean: 62.5 ). Median observation time was 12.8 months (95\% Cl: $11.2-13.9)$. The number of complete observations (deaths) in the afatinib-treated patients was 76 (28.5\%). The number of censored observations was 191 (71.5\%). Median OS was 22.8 months $(95 \% \mathrm{Cl}$ : 19.2-27.1). The probability of surviving 12 months was $75.4 \%$, 24 months $-41.9 \%$.

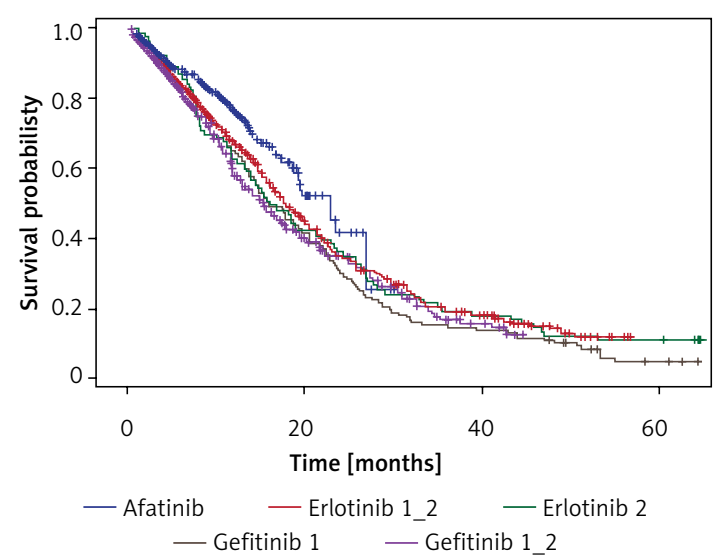

Figure 1. Kaplan-Meier estimation of overall survival for C34.\% patients subject to erlotinib, gefitinib or afatinib therapy
The number of patients in the first erlotinib subgroup ("erlotinib 2" label in Figure 1) was 83; 29 (34.9\%) were males, and 54 (65.1\%) were females. Median males' age was $60.5 \pm 8.1$ years (mean: 62.6); median females' age was $62 \pm 9.7$ years (mean: 62.7). Median observation time was 64.3 months ( $95 \% \mathrm{Cl}: 60.4-64.6)$. In this subgroup, there were $74(89.2 \%)$ complete observations (deaths) and 9 (10.8\%) censored observations. Median OS was 16 months (95\% Cl: 13.2-22.9). Probability of surviving 12 months was $61.5 \%$, 24 months - 35\%, 36 months - 19.3\%.

Six hundred twenty-two patients were in the second erlotinib subgroup ("erlotinib 1_2" label in Figure 1); 203 (32.6\%) males and 419 (67.4\%) females. Median males' age was $63 \pm 10.8$ years (mean $=63.2)$; median females' age was $67 \pm 9.6$ years (mean $=66.3$ ). Median observation time was 33.3 months ( $95 \% \mathrm{Cl}: 31.2-37.6)$. In this subgroup, there were $400(64.3 \%)$ complete observations (deaths) and 222 (35.7\%) censored observations. Median OS was 17.8 months (95\% Cl: $16.4-19.7)$. Probability of surviving 12 months was $67.6 \%$, 24 months $-34.3 \%$, 36 months $-19.4 \%$.

The Japanese multicenter phase II study presented at ASCO 2018 showed the results of lowdose erlotinib for patients with frailty. In the group of 80 patients, median progression-free survival was 9.2 months, while median survival time and 1 -year survival rate were 26.3 months and $68.9 \%$, respectively [2].

In the Chilean single institution experience from Centro Internacional de Estudios Clinicos with erlotinib in EGFR mutation-positive metastatic nonsmall-cell lung cancer patients, also presented at ASCO 2018, progression-free survival was 17 months and median overall survival 19 months [3].

The first gefitinib subgroup ("gefitinib 1" label in Figure 1) consisted of 137 patients: 42 (30.7\%) males and 95 (69.3\%) females; median males' age was $64 \pm 10.4$ years (mean: 66.1); median females' age was $66 \pm 12.6$ years (mean: 64.4 ). Median observation time was 58.3 months $(95 \% \mathrm{Cl}$ : 49.462.5). There were 128 (93.4\%) complete observations (deaths) and 9 (6.6\%) censored observations. Median OS was 16 months (95\% Cl: 13.8-19.7). Probability of surviving 12 months was $64.2 \%$; 24 months $-29.2 \%$, 36 months $-14.6 \%$.

The second gefitinib subgroup ("gefitinib 1_2" label in Figure 1) consisted of 348 patients: 109 (31.3\%) males and 239 (68.7\%) females; median males' age was $66 \pm 10.5$ years (mean: 66.2 ); median females' age was $68 \pm 11.1$ years (mean: 67.6). Median observation time was 23.7 months $(95 \% \mathrm{Cl}$ : 20.7-28.7). The number of complete observations (deaths) was 208 (59.8\%), and the number of censored observations in this subgroup was 140 (40.2\%). Median OS was 15.5 months $(95 \% \mathrm{Cl}$ : 
Table I. Data on erlotinib, gefitinib or afatinib therapy

\begin{tabular}{|c|c|c|c|c|c|}
\hline Parameter & Afatinib & Erlotinib 2 & Erlotinib 1_2 & Gefitinib 1 & Gefitinib 1_2 \\
\hline No. of patients & 267 & 83 & 622 & 137 & 348 \\
\hline \multicolumn{6}{|l|}{ Sex, $n(\%):$} \\
\hline Male & $99(37.1)$ & $29(34.9)$ & $203(32.6)$ & $42(30.7)$ & $109(31.3)$ \\
\hline Female & $168(62.9)$ & $54(65.1)$ & $419(67.4)$ & $95(69.3)$ & $239(68.7)$ \\
\hline $\begin{array}{l}\text { No. of complete } \\
\text { observations (\%) }\end{array}$ & $76(28.5)$ & $74(89.2)$ & $400(64.3)$ & $128(93.4)$ & $208(59.8)$ \\
\hline $\begin{array}{l}\text { Median observation } \\
\text { time [months] }(95 \% \mathrm{Cl})\end{array}$ & $12.8(11.2-13.9)$ & $64.3(60.4-64.6)$ & $33.3(31.2-37.6)$ & $58.3(49.4-62.5)$ & $23.7(20.7-28.7)$ \\
\hline $\begin{array}{l}\text { Median overall survival } \\
\text { [months] }(95 \% \mathrm{Cl})\end{array}$ & $22.8(19.2-27.1)$ & $16(13.2-22.9)$ & $17.8(16.4-19.7)$ & $16(13.8-19.7)$ & $15.5(12.9-17.5)$ \\
\hline $\begin{array}{l}\text { Probability of surviving } \\
12 \text { months (\%) }\end{array}$ & 75.4 & 61.5 & 67.6 & 64.2 & 58.2 \\
\hline $\begin{array}{l}\text { Probability of surviving } \\
24 \text { months (\%) }\end{array}$ & 41.9 & 35 & 34.3 & 29.2 & 34.5 \\
\hline $\begin{array}{l}\text { Probability of surviving } \\
36 \text { months (\%) }\end{array}$ & Not reached & 19.3 & 19.4 & 14.6 & 16.8 \\
\hline
\end{tabular}

Table II. Patient age characteristics

\begin{tabular}{|c|c|c|c|c|c|}
\hline Age characteristics & Afatinib & Erlotinib 2 & Erlotinib 1_2 & Gefitinib 1 & Gefitinib 1_2 \\
\hline Median age & 64.00 & 61.00 & 66.00 & 35.00 & 67.00 \\
\hline Mean age & 62.18 & 62.65 & 65.34 & 48.86 & 67.15 \\
\hline $95 \% \mathrm{Cl}$ & $(61.08-63.27)$ & $(60.90-64.41)$ & $(64.74-65.93)$ & $(24.92-72.79)$ & $(66.25-68.05)$ \\
\hline SD & 11.56 & 9.16 & 10.10 & 25.88 & 10.95 \\
\hline$P$-value & $<0.0001$ & $<0.0001$ & $<0.0001$ & 0.0025 & $<0.0001$ \\
\hline $20-29$ & $0.75 \%$ & $0.00 \%$ & $0.37 \%$ & $0.00 \%$ & $0.37 \%$ \\
\hline $30-39$ & $4.12 \%$ & $0.00 \%$ & $4.12 \%$ & $1.50 \%$ & $1.12 \%$ \\
\hline $40-49$ & $9.36 \%$ & $1.87 \%$ & $14.61 \%$ & $4.12 \%$ & $7.87 \%$ \\
\hline $50-59$ & $24.72 \%$ & $11.61 \%$ & $46.82 \%$ & $11.99 \%$ & $22.85 \%$ \\
\hline $60-69$ & $42.32 \%$ & $10.11 \%$ & $97.75 \%$ & $14.23 \%$ & $48.31 \%$ \\
\hline $70-79$ & $22.10 \%$ & $8.24 \%$ & $67.42 \%$ & $17.23 \%$ & $39.33 \%$ \\
\hline
\end{tabular}

12.9-17.5). Probability of surviving 12 months was $58.2 \%$; 24 months - 34.5\%, 36 months - 16.8\%. The summary of the data on erlotinib, gefitinib and afatinib therapy can be found in Tables I and II.

\section{Discussion}

In patients with locally advanced, inoperative and not qualified for radical radiotherapy or chemoradiotherapy, or metastatic NSCLC with confirmed presence of an activating mutation in the EGFR gene, the basic therapeutic option is systemic treatment with low molecular weight EGFR receptor tyrosine kinase inhibitors. We presented the OS time data for all patients with lung cancer receiving afatinib, erlotinib or gefitinib reimbursed from public funds in Poland.
In Poland, the use of afatinib [4] in the $1^{\text {st }}$ line and gefitinib or erlotinib [5] in the $1^{\text {st }}$ or $2^{\text {nd }}$ line of therapy (after prior platinum compounds use) is allowed according to the DPs. These programmes apply to patients with locally advanced or metastatic, histologically or cytologically diagnosed adenocarcinoma, NSCLC with predominant adenocarcinoma, large-cell carcinoma or not otherwise specified non-small cell carcinoma (NOS), with an EGFR activating mutation. Inclusion and exclusion criteria for DPs are strict and limit the population compared to SmPC (e.g. good performance status and absence of comorbidities) [4, 5]. This makes the population of lung cancer patients treated with EGFR inhibitors in Poland more uniform and similar to cohorts participating in clinical studies. 
Initial studies assessing the use of EGFR receptor kinase inhibitors (gefitinib and erlotinib), which indicate their effectiveness in the treatment of NSCLC, were conducted in a population not selected for EGFR gene status. However, based on analyses of those studies, it was possible to find EGFR activating mutations as a molecular prediction factor, correlated strongly with a better outcome with low molecular weight EGFR receptor tyrosine kinase inhibitors.

The first randomised clinical study which compared the anti-EGFR therapy with chemotherapy, also in the sub-group of patients with tumours with an EGFR mutation present, was IPASS (Iressa Pan-Asia Study). The study was conducted in a population with the highest probability of the response: previously untreated patients of East Asian origin, with a stage IIIB/IV lung adenocarcinoma, who never smoked or were former light smokers. A significant benefit for PFS was observed in a subgroup of 261 patients with an activating EGFR gene mutation $(\mathrm{HR}=0.48 ; 95 \% \mathrm{Cl}$ : $0.36-0.64 ; p<0.001)[6,7]$.

Later on, the results of multiple subsequent studies assessing the use of gefitinib or erlotinib with stage IIIB/IV NSCLC patients with probable or confirmed presence of activating EGFR mutation were published: EURTAC (European Randomised Trial of Tarceva versus Chemotherapy) [8, 9], OPTIMAL [10, 11], NEJ002 (North East Japan 002) [12, 13], West Japan Thoracic Oncology Group (WJTOG3405 [14, 15] and ENSURE [16]. Most of them were not conducted in the Asian population. The median overall survival for patients treated with erlotinib in the $1^{\text {st }}$ line in the EUTRAC study (European population) was 22.9 months [9], in the OPTIMAL study (Asiatic population) 22.8 months [11], whereas in the ENSURE study (Asiatic population) it was 26.3 months [16]. The median OS of patients treated in the $1^{\text {st }}$ line with gefitinib was 27.7 months in the final analysis of the NEJOO2 study (Asiatic population) [13] and 34.8 months in the WJTOG3405 study (Asiatic population) [15]. These studies indicated a significant PFS benefit to patients treated with EGRF receptor tyrosine kinase inhibitors and a milder toxicity profile when compared to chemotherapy and ensured the important place for these drugs in the treatment of NSCLC patients with an activating EGRF gene mutation.

The efficacy of another anti-EGFR drug, afatinib (a selective, irreversible inhibitor of HER family tyrosine kinase receptors) in the $1^{\text {st }}$ line treatment of stage IIIB/IV lung adenocarcinoma was assessed in two phase III studies: LUX-Lung 3 and LUX-Lung 6. In the LUX-Lung 3 study ( $n=345$ patients), the median OS was 28.2 months $(95 \% \mathrm{Cl}$ : 24.6-33.6), and in the LUX-Lung 6 study $(n=364$ patients) it was 23.1 months ( $95 \%$ Cl: 20.4-27.3).
The subgroup analysis indicated longer median OS in patients with a deletion in the $19^{\text {th }}$ exon of the EGFR gene treated with afatinib compared to chemotherapy: 33.3 (95\% Cl: $26.8-41.5)$ vs. 31.4 months (95\% Cl: 24.2-35.3) respectively [17].

In the phase IIB LUX-lung 7 study, the effectiveness of the $1^{\text {st }}$ line therapies with afatinib or gefitinib was compared. Despite differences found with regards to progression-free survival (PFS) and the direct response rate benefiting afatinib, no significant difference in the overall survival was observed. After an observation period of approximately 43 months, the median OS for afatinib was 27.9 months and for gefitinib 24.5 months ( $H R=$ $0.86,95 \% \mathrm{Cl}: 0.66-1.12, p=0.2580)[18]$.

The survival of NSCLC patients treated with EGFR inhibitors outside of clinical studies was compared in a retrospective analysis conducted in a Japanese patient population: 147 patients treated with afatinib, erlotinib or gefitinib [19]. No significant differences were observed in PFS and OS between individual drugs. The median PFS and OS for afatinib, erlotinib and gefitinib were, respectively, 13.1 vs. 9.8 vs. 9.2 months and not achieved vs. 29.3 vs. 27.3 months [19]. The comparable efficiency of gefitinib and erlotinib with regards to OS was also established in 2017 by a meta-analysis including 17,621 patients from 8 randomised studies and 82 cohort studies ( $\mathrm{HR}=0.99,95 \% \mathrm{Cl}: 0.93-1.06)$ [20].

In the recently published analysis of patients receiving EGFR inhibitors in the $1^{\text {st }}$ or $2^{\text {nd }}$ line in 4 Dutch hospitals, median OS in patients with EGFR mutation was 720 days [21].

Median OS in the case of erlotinib use in 1st line treatment of stage IIIB/IV NSCLC patients with an EGFR mutation observed in phase III studies was approximately 22-27 months [18-20], 24-35 months for gefitinib [7, 13, 14], and 23-28 months for afatinib (33.3 months in a subgroup with exon 19 deletion) $[6,7]$. The number of patients receiving the drug given in these studies varied from 86 to 242 , and the largest population was treated with afatinib [22]. The results from the Japanese retrospective analysis are slightly better for erlotinib (29.3 months) and similar for gefitinib (27.3 months) compared to phase III studies.

The overall survival of patients treated with gefitinib or erlotinib assessed by us is shorter than observed either in phase III studies or the Japanese retrospective analysis. Moreover, in the case of erlotinib, it was even shorter than in the Japanese study involving only patients with frailty and the Chilean study including exclusively patients with metastases.

In the case of patients treated in Poland with erlotinib in the $1^{\text {st }}$ or $2^{\text {nd }}$ line, median OS is 17.8 months compared to 22-27 months, and 15.5 months vs. 24-35 months for gefitinib. 
The shorter survival in our group may result from the fact that a significant part of the population assessed received EGFR inhibitors in the $2^{\text {nd }}$, but not in the $1^{\text {st }}$ line. This explanation is additionally supported by the fact that the observed results of afatinib treatment (used in the $1^{\text {st }}$ line only) are close to those obtained in the clinical studies. The median OS was 22.8 vs. 23-28 months. Considering the results of subgroup analyses of LUX-Lung 3 and LUX-Lung 6 studies, which suggest longer survival of patients with EGFR exon 19 deletion, differences between our and clinical studies' data may also result from differences in the frequency of mutation types in the populations concerned, especially considering that most studies assessing the effectiveness of gefitinib and erlotinib were conducted in the Asiatic population [17].

The obvious restriction of our analysis is the lack of detailed data concerning patient characteristics, PFS, the frequency of direct responses and safety. Also, we were not able to calculate the proportion of patients with locally advanced vs. metastatic cancer. However, beyond any doubt, the major value of the study is the population-wide scope (practically all NSCLC patients who have received anti-EGFR drugs reimbursed by the public payer in Poland) and the long period of analysis.

In conclusion, financing of afatinib, erlotinib and gefitinib under DPs with specific inclusion criteria makes it possible to obtain OS results comparable to those described in phase III clinical trials for $1^{\text {st }}$ line treatment, enabling good access to innovative, expensive therapies for patients with advanced NSCLC with an EGFR activating mutation present. However, the lower overall survival rate of Polish patients compared to similar studies from other research centres suggests the need for deeper investigation of this issue.

\section{Conflict of interest}

The authors declare no conflict of interest.

\section{References}

1. Sharma D, Newman T, Aronow W. Lung cancer screening: history, current perspectives, and future directions. Arch Med Sci 2015; 11: 1033-43.

2. Yamada K, Miyamoto S, Azuma K, et al. A multicenter phase II study of low-dose erlotinib in frail patients with EGFR mutation-positive, non-small cell lung cancer: Thoracic Oncology Research Group (TORG) trial 1425. J Clin Oncol 2018; 36 Suppl; abstr 9063.

3. Aren O, Silva R, Cerda H, et al. Single institution experience with $75 \mathrm{mg}$ dose of erlotinib in Latin American patients with mutated metastatic non-small cell lung cancer. J Clin Oncol 2015; 33 Suppl.: abstr 8075.

4. Treatment of NSCLC with afatinib. Description of the drug programme in Poland. Accessed: March $6^{\text {th }} 2018$. http://onkologia online.pl/upload/obwieszczenie/2017. 12.21/b/b.63.pdf.
5. Treatment of NSCLC. Description of the drug programme in Poland. Accessed: March $6^{\text {th }}$ 2018. http:// onkologia-online.pl/upload/obwieszczenie/2017. 12.21/b/b.6.pdf .

6. Mok TS, Wu YL, Thongprasert S, et al. Gefitinib or carboplatin-paclitaxel in pulmonary adenocarcinoma. N Engl J Med 2009; 361: 947-57.

7. First Line IRESSA ${ }^{\text {TM }}$ Versus Carboplatin/Paclitaxel in Asia (IPASS) Clinical Trial Results. Accessed: March 6 2018. https://clinicaltrials.gov/ct2/show/results/NCT00322452? sect $=$ X01256

8. Rosell R, Carcereny E, Gervais R, et al. Erlotinib versus standard chemotherapy as first-line treatment for European patients with advanced EGFR mutation-positive nonsmall-cell lung cancer (EURTAC): a multicentre, open-label, randomised phase 3 trial. Lancet Oncol 2012; 13: 239-46.

9. Costa C, Molina MA, Drozdowskyj A, et al. The impact of EGFR T790M mutations and BIM mRNA expression on outcome in patients with EGFR-mutant NSCLC treated with erlotinib or chemotherapy in the randomized phase III EURTAC trial. Clin Cancer Res 2014; 20: 2001-10.

10. Zhou C, Wu YL, Chen G, et al. Erlotinib versus chemotherapy as first-line treatment for patients with advanced EGFR mutation-positive non-small-cell lung cancer (OPTIMAL, CTONG-0802): a multicentre, open-label, randomised, phase 3 study. Lancet Oncol 2011; 12: 735-42.

11. Zhou C, Wu YL, Chen G. Final overall survival results from a randomised, phase III study of erlotinib versus chemotherapy as first-line treatment of EGFR mutation-positive advanced non-small cell lung cancer (OPTIMAL, CTONG-0802). Ann Oncol 2015; 26: 1877-83.

12. Maemondo M, Inoue A, Kobayashi K, et al. Gefitinib or chemotherapy for non-small-cell lung cancer with mutated EGFR. N Engl J Med 2010; 362: 2380-8.

13. Inoue A, Kobayashi K, Maemondo M, et al. Updated overall survival results from a randomized phase III trial comparing gefitinib with carboplatin-paclitaxel for chemo-naïve non-small cell lung cancer with sensitive EGFR gene mutations (NEJ002). Ann Oncol 2013; 24: 54-9.

14. Mitsudomi T, Morita S, Yatabe Y, et al. Gefitinib versus cisplatin plus docetaxel in patients with non-small-cell lung cancer harbouring mutations of the epidermal growth factor receptor (WJTOG3405): an open label, randomised phase 3 trial. Lancet Oncol 2010; 11: 121-8.

15. Yoshioka H, Mitsudomi T, Morita S, et al. Final overall survival results of WJTOG 3405, a randomized phase 3 trial comparing gefitinib (G) with cisplatin plus docetaxel $(C D)$ as the first-line treatment for patients with non-small cell lung cancer (NSCLC) harboring mutations of the epidermal growth factor receptor (EGFR). Available at: http://ascopubs.org/doi/abs/10.1200/jco. 2014.32.15_suppl.8117.

16. Wu YL, Zhou C, Liam CK, et al. First-line erlotinib versus gemcitabine/cisplatin in patients with advanced EGFR mutation-positive non-small-cell lung cancer: analyses from the phase III, randomized, open-label, ENSURE study. Ann Oncol 2015; 26: 1883-9.

17. Yang JC, Wu YL, Schuler M. Afatinib versus cisplatinbased chemotherapy for EGFR mutation-positive lung adenocarcinoma (LUX-Lung 3 and LUX-Lung 6): analysis of overall survival data from two randomised, phase 3 trials. Lancet Oncol 2015; 16: 141-51.

18. Paz-Ares L, Tan EH, O'Byrne K, et al. Afatinib versus gefitinib in patients with EGFR mutation-positive advanced non-small-cell lung cancer: overall survival data from the phase IIb LUX-Lung 7 trial. Ann Oncol 2017; 28: 270-7. 
19. Fujiwara A, Yoshida M, Fujimoto H. A Retrospective comparison of the clinical efficacy of gefitinib, erlotinib and afatinib in Japanese patients with non-small cell lung cancer. Oncol Res 2018; 26: 1031-6.

20. Yang Z, Hackshaw A, Feng Q, et al. Comparison of gefitinib, erlotinib and afatinib in non-small cell lung cancer: a meta-analysis. Int J Cancer 2017; 140: 2805-19.

21. Sluga R, van den Borne BEEM, Roepman P, et al. Utilization of molecular testing and survival outcomes of treatment with first- or second-line tyrosine kinase inhibitors in advanced non-small cell lung cancer in a Dutch population. Anticancer Res 2018; 38: 393-400.

22. Sebastian M, Schmittel A, Reck M, et al. First-line treatment of EGFR-mutated non-small cell lung cancer: critical review on study methodology. Eur Respir Rev 2014; 23: 92-105. 


\section{Appendix}

\section{Qualification Criteria for Lung Cancer Drug Programme in Poland}

\section{A. Inclusion criteria for erlotinib as first-line therapy:}

1) histological or cytological diagnosis of adenocarcinoma (including subtypes) or non-small cell carcinoma with the predominance of adenocarcinoma or large cell carcinoma, or non-small cell carcinoma not otherwise specified (NOS);

2) locally advanced (stage III - except for cases where chemoradiotherapy, radiotherapy or surgical treatment is possible) or generalised (stage IV);

3) no prior pharmacological treatment of locally advanced or generalised NSCLC;

4) cancer is measurable;

5) in the case of a single change - localised outside of the area of prior irradiation;

6) presence of activating mutation of the EGFR gene confirmed:

7) response to treatment assessment according to the RECIST 1.1 criteria possible;

8) $\geq 18$ years of age;

9) 0-1 performance status according to the Zubrod-WHO or ECOG criteria;

10) absence of clinically significant co-morbidities as uncontrolled hypertension, unstable coronary disease, myocardial infarction in the last year, ventricular arrhythmias requiring treatment;

11) lack of metastases or signs of metastases' progression in the central nervous system after prior local treatment (surgery or radiotherapy), without neurological symptoms and the need of glucocorticosteroids dose increase during the month preceding enrolment to the program;

12) normal function of the hematopoietic system, allowing treatment in accordance with the current Summary of Product Characteristics (SmPC);

13) normal kidneys' function:

a) creatinine concentration $\leq 1.5 \times$ upper limit of normal (ULN),

b) creatinine clearance $\geq 45 \mathrm{ml} / \mathrm{min}$;

14) normal liver function:

a) bilirubin concentration $\leq 1.5 \times$ ULN,

b) activity of transaminases and alkaline phosphatase $\leq 3 \times$ ULN or $\leq 5 \times$ ULN in the case of hepatic metastases;

15) no contraindications as per SmPC;

16) the use of concurrent chemotherapy and other molecularly targeted drugs is excluded.

All above criteria must be met jointly.

B. Inclusion criteria for erlotinib as second-line therapy:

1) histological or cytological diagnosis of adenocarcinoma (including subtypes) or non-small cell carcinoma with the predominance of adenocarcinoma or large cell carcinoma, or non-small cell carcinoma not otherwise specified (NOS);

2) locally advanced (stage III - except for cases where chemoradiotherapy, radiotherapy or surgical treatment is possible) or generalised (stage IV);

3) cancer is measurable;

4) in the case of a single change - localised outside of the area of prior irradiation;

5) presence of activating mutation of the EGFR gene confirmed;

6) response to treatment assessment according to the RECIST 1.1 criteria possible;

7) $\geq 18$ years of age;

8) absence of clinically significant co-morbidities as uncontrolled hypertension, unstable coronary disease, myocardial infarction in the last year, ventricular arrhythmias requiring treatment;

9) progression of the disease after prior chemotherapy involving at least one treatment line;

10) earlier application of multi-drug chemotherapy with the use of platinum derivatives (in the case of patients over 70 years old - previous monotherapy);

11) previous chemotherapy ended at least 3-4 weeks before enrolment;

12) absence of prior chemotherapy side effects except for hair loss;

13) lack of metastases or signs of metastases' progression in the central nervous system after prior local treatment (surgery or radiotherapy), without neurological symptoms and the need of glucocorticosteroids dose increase during the month preceding enrolment to the program;

14) 0-1 performance status according to the Zubrod-WHO or ECOG criteria;

15) normal function of the hematopoietic system, allowing treatment in accordance with the current Summary of Product Characteristics (SmPC);

16) normal kidneys' function:

a) creatinine concentration $\leq 1.5 \times$ upper limit of normal (ULN),

b) creatinine clearance $\geq 45 \mathrm{ml} / \mathrm{min}$;

17) normal liver function:

a) bilirubin concentration $\leq 1.5 \times$ ULN, 
b) activity of transaminases and alkaline phosphatase $\leq 3 \times$ ULN or $\leq 5 \times$ ULN in the case of hepatic metastases;

18) no contraindications as per SmPC;

19) the use of concurrent chemotherapy and other molecularly targeted drugs is excluded.

All above criteria must be met jointly.

C. Inclusion criteria for gefitinib as first-line therapy:

1) histological or cytological diagnosis of adenocarcinoma (including subtypes) or non-small cell carcinoma with the predominance of adenocarcinoma or large cell carcinoma, or non-small cell carcinoma not otherwise specified (NOS);

2) presence of activating mutation of the EGFR gene confirmed;

3) locally advanced (stage III - except for cases where chemoradiotherapy, radiotherapy or surgical treatment is possible) or generalized (stage IV);

4) response to treatment assessment in medical imaging according to the RECIST 1.1 criteria possible;

5) lack of metastases or signs of metastases' progression in the central nervous system after prior local treatment (surgery or radiotherapy), without neurological symptoms and the need of glucocorticosteroids dose increase during the month preceding enrolment to the program;

6) $\geq 18$ years of age;

7) 0-1 performance status according to WHO or ECOG criteria;

8) absence of clinically significant co-morbidities as uncontrolled hypertension, unstable coronary disease, myocardial infarction in the last year, ventricular arrhythmias requiring treatment;

9) normal function of the hematopoietic system, allowing treatment in accordance with the current Summary of Product Characteristics (SmPC);

10) normal kidneys' function (creatinine concentration $\leq 1.5 \times$ upper limit of normal (ULN));

11) normal liver function:

a) bilirubin concentration $\leq 1.5 \times$ ULN,

b) activity of transaminases and alkaline phosphatase $\leq 3 \times$ ULN or $\leq 5 \times$ ULN in the case of hepatic metastases;

12) no contraindications as per SmPC;

13) the use of concurrent chemotherapy and other molecularly targeted drugs is excluded;

14) no prior pharmacological treatment of locally advanced or generalized NSCLC;

15) no other malignancies treated with a palliative assumption (regardless of the response obtained) or failure to obtain a complete response in the case of cancer treated with a radical assumption;

16) response to treatment assessment according to the RECIST 1.1 criteria possible or the presence of countable non-measurable changes.

All above criteria must be met jointly.

D. Inclusion criteria for gefitinib as second-line therapy:

1) histological or cytological diagnosis of adenocarcinoma (including subtypes) or non-small cell carcinoma with predominance of adenocarcinoma or large cell carcinoma, or non-small cell carcinoma not otherwise specified (NOS);

2) presence of activating mutation of the EGFR gene confirmed;

3) locally advanced (stage III - except of cases where chemoradiotherapy, radiotherapy or surgical treatment is possible) or generalized (stage IV);

4) response to treatment assessment in medical imaging according to the RECIST 1.1 criteria possible;

5) lack of metastases or signs of metastases' progression in the central nervous system after prior local treatment (surgery or radiotherapy), without neurological symptoms and the need of glucocorticosteroids dose increase during the month preceding enrolment to the program;

6) $\geq 18$ years of age;

7) 0-1 performance status according to WHO or ECOG criteria;

8) absence of clinically significant co-morbidities as uncontrolled hypertension, unstable coronary disease, myocardial infarction in the last year, ventricular arrhythmias requiring treatment;

9) normal function of the hematopoietic system, allowing treatment in accordance with the current Summary of Product Characteristics (SmPC);

10) normal kidneys' function (creatinine concentration $\leq 1.5 \times$ upper limit of normal (ULN));

11) normal liver function:

a) bilirubin concentration $\leq 1.5 \times$ ULN,

b) activity of transaminases and alkaline phosphatase $\leq 3 \times$ ULN or $\leq 5 \times$ ULN in the case of hepatic metastases;

12) no contraindications as per SmPC;

13) no prior use of EGFR tyrosine kinase inhibitors;

14) the use of concurrent chemotherapy and other molecularly targeted drugs is excluded;

15) confirmation of disease progression after prior chemotherapy (at least one treatment line); 
16) use of pre-existing multi-drug chemotherapy with the use of platinum derivatives (in the case of patients $>70$ years old - previous monotherapy) - the required period from the end of prior chemotherapy - at least 4 weeks;

17) response to treatment assessment according to the RECIST 1.1 criteria possible, or the presence of countable non-measurable changes;

18) absence of prior chemotherapy side effects except of hair loss;

19) no other malignancies treated with a palliative assumption (regardless of the response obtained) or failure to obtain a complete response in the case of cancer treated with a radical assumption.

All above criteria must be met jointly.

E. Inclusion criteria for afatinib as first-line therapy:

1) histological or cytological diagnosis of adenocarcinoma (including subtypes) or non-small cell carcinoma with predominance of adenocarcinoma or large cell carcinoma, or non-small cell carcinoma not otherwise specified (NOS);

2) presence of activating mutation of the EGFR gene confirmed;

3) locally advanced (stage III - except of cases where chemoradiotherapy, radiotherapy or surgical treatment is possible) or generalized (stage IV);

4) no prior pharmacological treatment of generalized or locally advanced NSCLC (excluding adjuvant therapy):

5) response to treatment assessment according to the RECIST 1.1 criteria possible. If the primary lung tumour does not exist (lung resection or lung cancer without primary tumour), it is necessary to show in imaging examinations measurable metastatic changes in or countable non-measurable changes;

6) in the case of a single change - localised outside of the area of prior irradiation;

7) response to treatment assessment in medical imaging according to the RECIST 1.1 criteria possible;

8) $\geq 18$ years of age;

9) 0-1 performance status according to the WHO criteria;

10) absence of clinically significant co-morbidities as uncontrolled hypertension, unstable coronary disease, myocardial infarction in the last year, ventricular arrhythmias requiring treatment;

11) lack of metastases or signs of metastases' progression in the central nervous system after prior local treatment (surgery or radiotherapy), without neurological symptoms and the need of glucocorticosteroids dose increase during the month preceding enrolment to the programme;

12) normal function of the hematopoietic system, allowing treatment in accordance with the current Summary of Product Characteristics (SmPC);

13) normal kidneys' function:

a) creatinine concentration $\leq 1.5 \times$ upper limit of normal (ULN),

b) creatinine clearance $\geq 45 \mathrm{ml} / \mathrm{min}$;

14) normal liver function:

a) bilirubin concentration $\leq 1.5 \times$ ULN,

b) activity of transaminases and alkaline phosphatase $\leq 3 \times$ ULN or $\leq 5 \times$ ULN in the case of hepatic metastases;

15) no contraindications as per SmPC;

16) the use of concurrent chemotherapy and other molecularly targeted drugs is excluded.

All above criteria must be met jointly.

F. Exclusion criteria:

1) progression of the disease according to the criteria of the RECIST 1.1:

a) an increase of existing changes by at least $20 \%$ or

b) the appearance of at least one new change - confirmed in a physical examination or imaging;

2) deterioration of the patient's condition due to cancer, without progression confirmed by physical examination or imaging;

3) occurrence of clinically significant treatment toxicity or occurrence of at least one life-threatening adverse event according to the CTC-AE classification criteria in version 4.03 (Common Terminology Criteria for Adverse Events - version 4.03);

4) occurrence of recurrent or unacceptable toxicity of treatment in grade 3 or 4 according to CTC-AE classification criteria in version 4.03 (resumption of treatment possible after resolution of toxicity or reduction to grade 1 or 2 according to CTC-AE classification criteria in the version 4.03);

5) hypersensitivity to the drug or any excipient;

6) decrease of performance status to 3-4 (2, 3, 4 if using erlotinib and gefitinib) according to WHO or ECOG criteria, or to $2-4$ according to WHO criteria for afatinib;

7) interruption of taking erlotinib or gefitinib lasting longer than 3 weeks, which was caused by an adverse effect of treatment;

8) deterioration of the quality of life of significant importance according to the doctor's assessment;

9) resignation of the patient - withdrawal of consent for participation in the program. 